\title{
Timepix3 detector network for nuclear waste monitoring
}

\author{
Bartolomej Biskup ${ }^{1}$, Benedikt Bergmann ${ }^{1}$, Pavel Broulim², Petr Burian ${ }^{1,2}$, Milan Malich ${ }^{1}$, Petr \\ Manek $^{1,3}$, Lukas Meduna ${ }^{1,2}$, Yesid Mora ${ }^{1}$, Martin Pichotka ${ }^{1,4}$, Lukas Pusman ${ }^{2}$, Peter Rubovic ${ }^{1}$, Tomas \\ Slavicek $^{1}$, and Petr Smolyanskiy ${ }^{1}$ \\ ${ }^{1}$ Institute of Experimental and Applied Physics, Czech Technical University in Prague, Czech \\ Republic \\ ${ }^{2}$ Faculty of Electrical Engineering, University of West Bohemia, Czech Republic \\ ${ }^{3}$ Department of Physics and Astronomy, University College London, United Kingdom \\ ${ }^{4}$ Clinic for Nuclear Medicine, University hospital Freiburg, Germany \\ Bartolomej.Biskup@utef.cvut.cz
}

\begin{abstract}
In the framework of the MICADO project we proposed an active long-term nuclear waste monitoring system based on Timepix3 technology. Particle detectors based on Timepix 3 are capable of particle type discrimination and provide spectrometric information in combination with precise timing information. To maximize the versatility of the setup, the Timepix 3 detectors employed are equipped with a range of different sensors. To enable measurement of neutrons, two quadrants of the detectors are covered by neutron converters, allowing detection of thermal neutron by capture in ${ }^{6} \mathrm{Li}$, as well as detection of fast neutrons through recoil protons generated in a polyethylene converter. Within this project a new housing for the detector unit and the readout was developed, with the network currently comprising of 9 such detectors. A specifically developed in-house software handles simultaneous operation of all devices, cluster analysis and data visualization. Energy calibration was performed using an X-ray tube with several fluorescence foils as well as photons from an ${ }^{241} \mathrm{Am}$ source. The neutron and gamma/Xray detection efficiency calibration was carried out at the Czech Metrology Institute and the results are presented here. Tests on simultaneous operation of all 9 devices were performed using uraninite and ${ }^{241} \mathrm{Am}$ sources.
\end{abstract}

Keywords - Timepix3 detector, nuclear waste monitoring, neutron detection.

\section{INTRODUCTION}

$\mathrm{T}$ HE Horizon 2020 funded MICADO [1] project (Measurement and Instrumentation for Cleaning and Decommissioning Operations) aims at proposing a costeffective solution for non-destructive characterization of nuclear waste, implementing a digitization process that could become a referenced standard facilitating and harmonizing the methodology used for the in-field Waste Management and Dismantling \& Decommissioning operations.

Distributed networks of hybrid semiconductor pixelated detectors (HPDs) have proven their efficiency and robustness in characterization of mixed radiation fields in various long- term investigations. In particular the ATLAS TPX network [2,3] has provided valuable data on particle flux within the ATLAS cavity at LHC since 2015. In 2018 this network, which was originally based on the Timepix 1 ASIC, has been amended by 4 Timpix $3[4,5]$ devices, resulting in improved particle discrimination capabilities as well as improved energetic and temporal resolution.

For this reason, in the framework of the MICADO project, we propose a long-term nuclear waste monitoring system based on Timepix3 technology as an alternative/complementary solution to the conventionally used detectors.

\section{TIMEPIX3 NETWORK}

Timepix 3 HPDs are capable of efficient particle discrimination and provide spectrometric information, in parallel with nanosecond resolution timing information. The active area of the sensor of $1.98 \mathrm{~cm}^{2}$ is formed by $256 \times 256$ pixels with a pixel pitch of $55 \mu \mathrm{m}$.

The particle flux encountered by these detectors, depending on nuclear waste composition, volume, geometry and the distance between the waste packages and detectors, is expected to cover a challenging range and to be composed of a mixed radiation field of ionizing and non-ionizing radiation. Moreover, in some cases, part of the detectors will be attached directly to the waste drums, while others are fixed to the storage walls. To maximize the versatility of the setup, the Timepix 3 detectors employed are equipped with a range of different sensors, namely Si sensors with thicknesses of 100, 300, and $500 \mu \mathrm{m}$, as well as CdTe sensors with a thickness of $1 \mathrm{~mm}$. The network currently comprises of 9 detectors.

To enable measurement of neutrons, two quadrants of the silicon detectors are covered by neutron converters. Thermal and epithermal neutrons are detected below a ${ }^{6} \mathrm{LiF}$ foil through products of a ${ }^{6} \mathrm{Li}(\mathrm{n}, \alpha)^{3} \mathrm{H}$ reaction and fast neutrons are detected through recoil protons under a polyethylene (PE) layer. In addition, an aluminum foil covering half of the sensor acts as an energy threshold. A schematic drawing of the converter structure together with the sensor, bump-bonds, and readout chip is shown in Fig. 1a. Since cadmium has a significant thermal 
neutron capture cross section, no convertors were used in CdTe equipped detectors. Katherine [6], an Ethernet Embedded Readout Interface for Timepix3, allows to operate our detectors via UDP protocol in distributed networks using standard networking hardware.

Within the MICADO project, a new housing for the readout and detector unit (Fig. lb.), optimized towards this specific task, was developed.

(a)
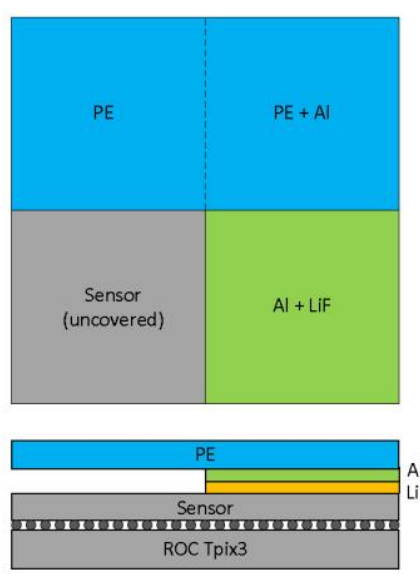

Fig. 1. (a) Structure of the converter (top and side view). (b) Housing of the Timepix 3 detector and Katherine readout with the sensor window and the filterholder in the upper part. Housing dimensions are $13 \mathrm{~cm} \times 20.4 \mathrm{~cm} \times 3 \mathrm{~cm}$.

A new plugin-based software, MM Track Lab, was developed in-house for data acquisition and processing. A specific MICADO detector network plugin handles simultaneous operation of multiple devices. Clustering of pixels, event analysis and data visualization (as visible in Fig. 2) is performed in real-time. An automated warning system, giving detailed characteristics of the potential nuclear hazard in case certain thresholds are surpassed, is integrated here as well.

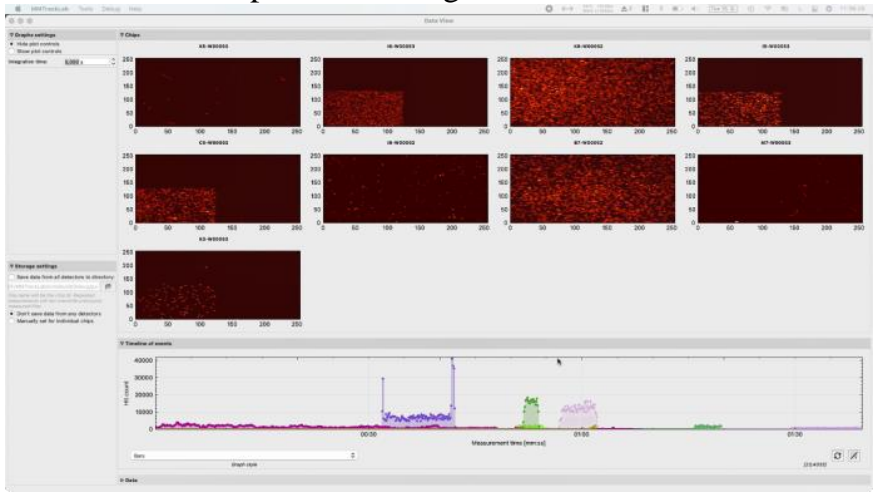

Fig. 2. Graphical user interface of the MM Track Lab MICADO detector network plugin. Individual matrices for all nine detectors measuring simultaneously are in the top part and the common timeline represents the number of events.

\section{CALIBRATION AND MEASUREMENTS}

A pixel-by-pixel energy calibration was performed using an
X-ray tube with several fluorescence foils as well as photons from an ${ }^{241} \mathrm{Am}$ source. An ${ }^{241} \mathrm{Am}$ source was used also for the time-walk calibration [7].

The neutron and gamma/X-ray detection efficiency calibration was carried out at the Czech Metrology Institute [8] using well defined radiation sources and reference fields.

Thermal neutrons were measured for each detector separately using neutrons from a ${ }^{238} \mathrm{PuBe}$ source, moderated in a graphite pile. Fluence at the detector position was $\left(2.92 \cdot 10^{4} \pm 1.80 \%\right) \mathrm{cm}^{-2} \cdot \mathrm{s}^{-1}$, corresponding to an ambient dose equivalent $\left(H^{*}(10)\right)$ of $(1.75 \pm 1.80 \%) \mathrm{mSv} / \mathrm{h}$. Sufficient statistics was reached for a measurement duration of $10 \mathrm{~min} /$ detector for $\mathrm{Si}$ sensors and $5 \mathrm{~min}$ for CdTe. For a fast neutron calibration, ${ }^{252} \mathrm{Cf}$ (ISO 8529-1) and ${ }^{241} \mathrm{AmBe}$ (ISO 8529-1) reference sources were used. A four-detector holder visible in Fig. 3 was used for this and most of the following measurements for efficiency. The ${ }^{241} \mathrm{AmBe}$ source was shielded by a lead capsule to minimize gamma background. Neutron fluences at the holder central positions were $\left(4.49 \cdot 10^{3} \pm 1.30 \%\right) \mathrm{cm}^{-2} \cdot \mathrm{s}^{-1}$ and $\left(4.32 \cdot 10^{3} \pm 1.50 \%\right) \mathrm{cm}^{-2} \cdot \mathrm{s}^{-1}$ for the ${ }^{241} \mathrm{AmBe}$ and ${ }^{252} \mathrm{Cf}$ sources, respectively, corresponding to ambient dose equivalents of $(6.32 \pm 2.40 \%) \mathrm{mSv} / \mathrm{h}$ and $(5.99$ $\pm 1.80 \%) \mathrm{mSv} / \mathrm{h}$. While the ${ }^{252} \mathrm{Cf}$ source can be approximated as a point-like source, the actual shape of the ${ }^{241} \mathrm{AmBe}$ must be considered when estimating systematic errors. Standard fast neutron measurement times were between $18 \mathrm{~min}$ and $90 \mathrm{~min}$. Several control measurements were executed overnight.

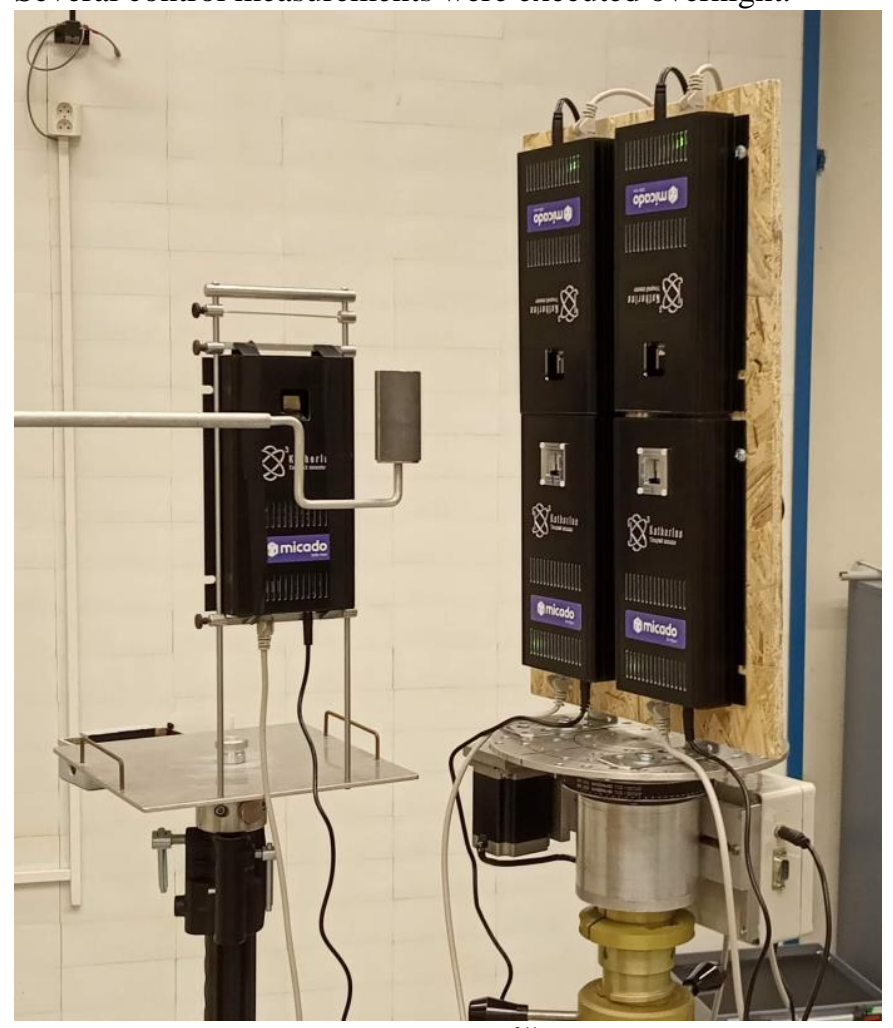

Fig. 3. Fast neutron calibration setup with an ${ }^{241} \mathrm{AmBe}$ source and five detectors operated simultaneously.

Photon calibration was conducted using gamma rays from ${ }^{60} \mathrm{Co}$ (with a mean energy $E_{\text {mean }}$ of $1174 \mathrm{keV}$ ) and ${ }^{137} \mathrm{Cs}$ $\left(E_{\text {mean }}=556 \mathrm{keV}\right)$ and standard X-ray beams N60 
$\left(E_{\text {mean }}=48 \mathrm{keV}\right), \quad \mathrm{N} 150 \quad\left(E_{\text {mean }}=118 \mathrm{keV}\right), \quad$ and $\quad \mathrm{N} 300$ $\left(E_{\text {mean }}=250 \mathrm{keV}\right)$. For each silicon detector, we collected $1 \mathrm{mSv}$ during a $6 \mathrm{~min}$ measurement for ${ }^{60} \mathrm{Co}$ and all X-rays and during $3 \mathrm{~min}$ for ${ }^{137} \mathrm{Cs}$. While for higher energies of ${ }^{60} \mathrm{Co}$ we roughly reached a data transfer limit of the CdTe setup with a $15 \mathrm{~m}$ Ethernet cable, for lower energy sources $\left({ }^{137} \mathrm{Cs}\right.$ at $20 \mathrm{mSv} / \mathrm{h}$ and X-rays at $10 \mathrm{mSv} / \mathrm{h}$ ) the data rate was so high that the system stopped working. Therefore, in order to avoid bandwidth problems and to reduce the overall amount of data taken to a reasonable amount, for the CdTe detectors we had to set up a lower beam intensity and decrease measurement time. With the new settings resulting in a collection of only $0.031 \mathrm{mSv}$, each output file still occupies several gigabytes. An angle scan was performed for one $300 \mu \mathrm{m} \mathrm{Si} \mathrm{detector} \mathrm{from}-90^{\circ}$ to $+90^{\circ}$ by $10^{\circ}$ rotation for the gamma sources and from $0^{\circ}$ to $+90^{\circ}$ by $15^{\circ}$ for the X-ray beams.

\section{RESUlTs}

To determine neutron fluences in the real environment, we must assess the neutron conversion efficiencies for each detector. The thermal and fast neutrons are observed below the corresponding neutron converters through the products of the abovementioned neutron reactions as roundish pixel clusters, so called "heavy blobs". By applying cuts based on the shape and deposited energy of the respective clusters, we can filter out most of the background, as depicted in Fig. $4 \mathrm{a}$ vs. Fig. 4b. Since the fast neutrons interact also directly in the silicon material, the heavy blobs are visible in the whole sensor area. The stronger signal below the $\mathrm{PE}+\mathrm{Al}$ quadrant in Fig. $4 \mathrm{c}$ suggests that the neutron spectrum is harder than the one in Fig. $4 \mathrm{~d}$. Such that we can distinguish between the signal from ${ }^{241} \mathrm{AmBe}$ with a mean energy of $4.16 \mathrm{MeV}$ and from ${ }^{252} \mathrm{Cf}$ with a mean energy of 2.13 $\mathrm{MeV}$.
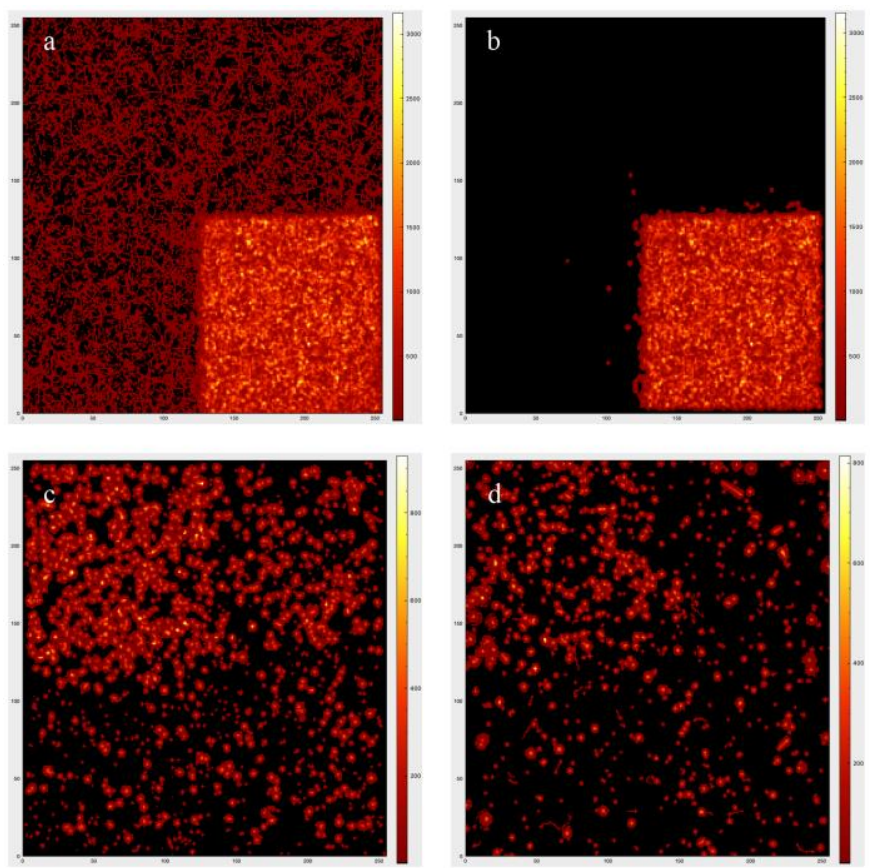

Fig. 4. (a) Integration window of thermal neutron measurement without any cluster cuts. (b) The same integration window as (a) but after cuts were applied. Almost no signal outside the LiF region. (c) Measurements with AmBe source after cluster cuts. More signal visible below $\mathrm{PE}$ and $\mathrm{PE}+\mathrm{Al}$ regions. (d) Measurements with Cf source after cluster cuts. Dominant PE region.

The neutron detection efficiencies can be calculated using

$$
\varepsilon_{A}=\frac{\frac{N_{A}}{S_{A}}-\frac{N_{S i}}{S_{S i}}}{\emptyset \cdot t},
$$

where $N_{A}$ and $N_{S i}$ are numbers of clusters detected during the measurement time $t$ in a region A (i.e., below ${ }^{6} \mathrm{LiF}$ for thermal neutrons and below PE for fast neutron measurement) and in the uncovered region, respectively. $S_{A}$ and $S_{S i}$ are the areas corresponding to these regions, and $\Phi$ is the incident neutron fluence. The conversion efficiencies for silicon detectors are listed in Table I. The probability of registering a thermal neutron is slightly above $1 \%$ for all detectors, while for fast neutrons it is more than factor of ten lower for the ${ }^{241} \mathrm{AmBe}$ source and another factor of 4-13 lower for the ${ }^{252} \mathrm{Cf}$ source. The resulting errors below $5 \%$ for the thermal neutron detection efficiency and below $15 \%$ for fast neutron efficiency are dominated by systematics (mainly geometry and position uncertainties) and are not listed individually.

TABLE I

NEUTRON CONVERSION EFFICIENCY

\begin{tabular}{|c|c|c|c|}
\hline Detector & $\varepsilon_{\mathrm{Li}(\text { thermal } \mathrm{n})}$ & $\varepsilon_{\mathrm{PE}(\mathrm{AmBe})}$ & $\varepsilon_{\mathrm{PE}(\mathrm{Cf})}$ \\
\hline I6 $100 \mu \mathrm{m} \mathrm{Si}$ & $1.30 \%$ & $0.080 \%$ & $0.008 \%$ \\
\hline $\mathrm{M7} 100 \mu \mathrm{m} \mathrm{Si}$ & $1.28 \%$ & $0.080 \%$ & $0.006 \%$ \\
\hline 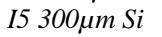 & $1.41 \%$ & $0.088 \%$ & $0.020 \%$ \\
\hline 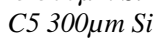 & $1.29 \%$ & $0.079 \%$ & $0.019 \%$ \\
\hline K3 $500 \mu m \mathrm{Si}$ & $1.04 \%$ & $0.093 \%$ & $0.020 \%$ \\
\hline 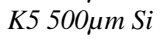 & $1.16 \%$ & $0.098 \%$ & $0.020 \%$ \\
\hline
\end{tabular}

Efficiency of neutron detection in the Si sensors through the products of neutron reactions with the converter materials (i.e., ${ }^{6} \mathrm{LiF}$ and $\mathrm{PE}$ ).

While these efficiencies can be directly used to calculate neutron fluences in real radiation environments (using the same cluster cuts as for the calibration), in case of CdTe sensors it is not a straightforward procedure. Table II summarizes only a ratio between number of impacted neutrons and number of clusters induced in CdTe by neutrons and accompanying photons. These data are nevertheless used to train our algorithms and still give a valuable information together with the cluster deposited energies, and other cluster features.

TABLE II

NeUtRon AND PHoton INDUCED Clusters in CDTE PER IMPACTED NEUTRON

\begin{tabular}{llll}
\hline \hline \multicolumn{1}{c}{ Detector } & $\mathrm{n}_{\mathrm{cl}} / \mathrm{n}_{\text {th }}$ & $\mathrm{n}_{\mathrm{cl}} / \mathrm{n}_{\mathrm{AmBe}}$ & $\mathrm{n}_{\mathrm{cl}} / \mathrm{n}_{\mathrm{Cf}}$ \\
\hline B7 1mm CdTe & 0.15 & 0.93 & 0.95 \\
I8 1mm CdTe & 0.15 & 1.08 & 0.86 \\
K8 1mm CdTe & 0.16 & 0.96 & 1.00 \\
\hline \hline
\end{tabular}

Number of clusters caused by neutrons and accompanying photons inside the CdTe sensor material per number of impacted neutrons.

The neutron energy spectra for the three calibration sources inside the CdTe detector are plotted as Time over Threshold in Fig. 5a. Fig. 5b and Fig. 5c show a comparison of Time over Threshold ${ }^{60} \mathrm{Co}$ and ${ }^{137} \mathrm{Cs}$ spectra between the CdTe and $\mathrm{Si}$ detectors. These spectra can be comprehended as a justification 
for using CdTe alongside the Si sensors, as even the thickest Si sensor employed in the network is not capable of absorbing the high-energy photons of ${ }^{60} \mathrm{Co}$ (at $1.17 \mathrm{MeV}$ and $1.33 \mathrm{MeV}$ ).

Number of clusters per ambient dose equivalent $H^{*}(10)$ was studied as a function of the photon source energy for all detectors. Fig. 6 shows such a dependency for each of the four sensor types used in our network. The behavior of all detectors is as predicted and follows the same tendency.
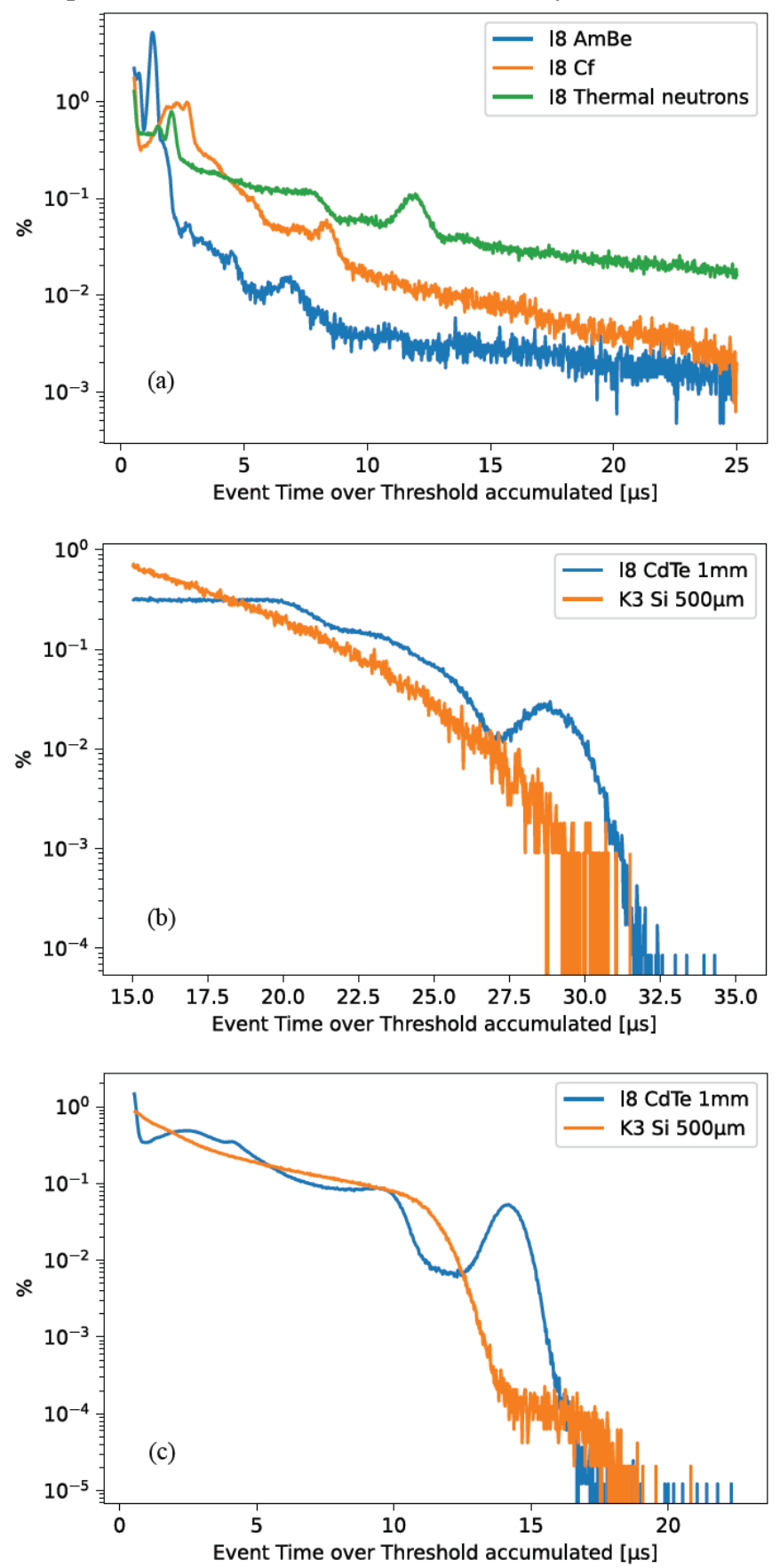

Fig. 5. Time over Threshold spectra of (a) three calibration neutron sources in the CdTe detector, (b) ${ }^{60} \mathrm{Co}$ in the CdTe and Si detectors, and (c) ${ }^{137} \mathrm{Cs}$ in the CdTe and Si detectors.

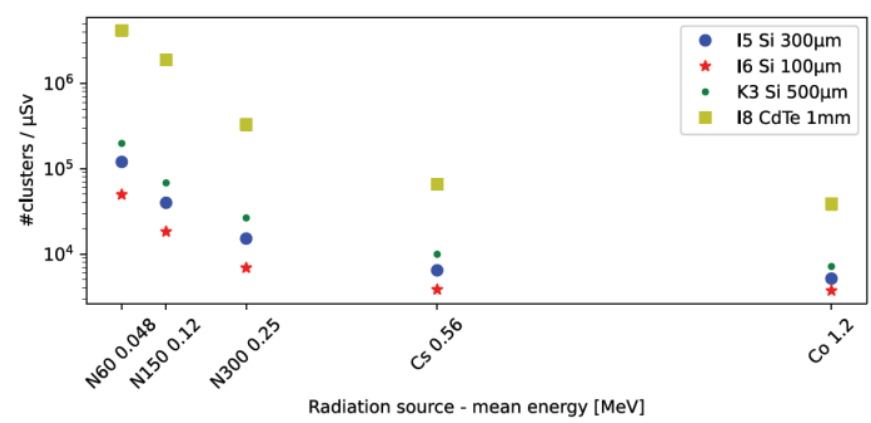

Fig. 6. Number of clusters per ambient dose equivalent as a function of the photon source energy.

Long-term simultaneous operation testing of all nine devices was performed using uraninite and ${ }^{241} \mathrm{Am}$ sources and exhibited good stability of the system.

\section{V.CONCLUSIONS}

In the framework of the MICADO project we devised an active long-term nuclear waste monitoring system, based on Timepix 3 technology. To maximize the versatility of the network, 9 detectors, featuring four different sensor and neutron converter configurations, were integrated. A specifically developed in-house software handles simultaneous operation of all these devices, as well as cluster analysis and data visualization. Calibration, characterization and testing of the system exhibited high particle discrimination efficiency and sufficient stability for real world application. Measurements on Radwaste sites are currently being prepared.

\section{ACKNOWLEDGMENT}

This work was funded within the framework of European Union's Horizon 2020 research and innovation programme under grant agreement No 847641, project MICADO (Measurement and Instrumentation for Cleaning And Decommissioning Operations).

\section{REFERENCES}

[1] MICADO project official website. [Online]. Available: https://www.micado-project.eu/. Accessed on: August 10, 2021

[2] B. Bergmann, I. Caicedo, C. Leroy, S. Pospisil and Z. Vykydal, "ATLAS-TPX: a two-layer pixel detector setup for neutron detection and radiation field characterization," JINST, vol. 11, Oct. 2016, Art no. P10002.

[3] B. Bergmann, T. Billoud, C. Leroy and S. Pospisil, "Characterization of the Radiation Field in the ATLAS Experiment With Timepix Detectors," IEEE Trans. Nucl. Sci., vol. 66, no. 7, pp. 1861-1869, July 2019.

[4] T. Poikela et al., "Timepix3: a 65K channel hybrid pixel readout chip with simultaneous ToA/ToT and sparse readout," JINST, vol. 9, May 2014, Art no. C05013.

[5] P. Burian, P. Broulim, B. Bergmann, V. Georgiev, S. Pospisil, L. Pusman and J. Zich, "Timepix3 detector network at ATLAS experiment," JINST, vol. 13, Nov. 2018, Art no. C11024.

[6] P. Burian, P. Broulim, M. Jara, V. Georgiev and B. Bergmann, "Katherine: Ethernet Embedded Readout Interface for Timepix3," JINST, vol. 12, Nov. 2017, Art no. C11001.

[7] D. Turecek, J. Jakubek and P. Soukup, "USB 3.0 readout and time-walk correction method for Timepix3 detector," JINST, vol. 11, Dec. 2016, Art no. C12065.

[8] Czech Metrology Institute website. [Online]. Available: https://www.cmi.cz/?lang=2\&language=en. Accessed on: August 10, 2021. 